\title{
Near-infrared imaging of brain tumors using the Tumor Paint BLZ-100 to achieve near-complete resection of brain tumors
}

\author{
Pramod V. Butte, M.B.B.S., Ph.D., ${ }^{1}$ Adam Mamelak, M.D., ${ }^{1}$ Julia Parrish-Novak, Ph.D., 2 \\ Doniel Drazin, M.D., ${ }^{1}$ Faris Shweikeh, B.S., ${ }^{1}$ Pallavi R. Gangalum, Ph.D., ${ }^{1}$ \\ Alexandra Chesnokova, M.D., ${ }^{1}$ Julia Y. LJubimova, M.D., Ph.D., ${ }^{1}$ \\ AND KeITH BLACK, M.D. ${ }^{1}$
}

${ }^{1}$ Department of Neurosurgery, Cedars-Sinai Medical Center, Los Angeles, California; and ${ }^{2}$ Blaze Bioscience, Inc., Seattle, Washington

Object. The intraoperative clear delineation between brain tumor and normal tissue in real time is required to ensure near-complete resection without damaging the nearby eloquent brain. Tumor Paint BLZ-100, a tumor ligand chlorotoxin (CTX) conjugated to indocyanine green (ICG), has shown potential to be a targeted contrast agent. There are many infrared imaging systems in use, but they are not optimized to the low concentration and amount of ICG. The authors present a novel proof-of-concept near-infrared (NIR) imaging system using a standard charge-coupled device (CCD) camera for visualizing low levels of ICG attached to the tumors. This system is small, inexpensive, and sensitive. The imaging system uses a narrow-band laser at $785 \mathrm{~nm}$ and a notch filter in front of the sensor at the band. The camera is a 2-CCD camera, which uses identical CCDs for both visible and NIR light.

Methods. The NIR system is tested with serial dilution of BLZ-100 from $1 \mu \mathrm{M}$ to $50 \mathrm{pM}$ in $5 \%$ Intralipid solution while the excitation energy is varied from 5 to $40 \mathrm{~mW} / \mathrm{cm}^{2}$. The analog gain of the CCD was changed from 0 , 6 , and $12 \mathrm{~dB}$ to determine the signal-to-noise ratio. In addition to the Intralipid solution, BLZ-100 was injected 48 hours before euthanizing the mice that were implanted with the human glioma cell line. The brain was removed and imaged using the NIR imaging system.

Results. The authors' results show that the NIR imaging system using a standard CCD is able to visualize the ICG down to $50 \mathrm{nM}$ of concentration with a high signal-to-noise ratio. The preliminary experiment on human glioma implanted in mouse brains demonstrated that BLZ-100 has a high affinity for glioma compared with normal brain tissue. Additionally, the results show that NIR excitation is able to penetrate deeply and has a potential to visualize metastatic lesions that are separate from the main tumor.

Conclusions. The authors have seen that BLZ-100 has a very high affinity toward human gliomas. They also describe a small, cost-effective, and sensitive NIR system for visualizing brain tumors tagged using BLZ-100. The authors hope that the use of BLZ-100 along with NIR imaging will be useful to delineate the brain tumors in real time and assist surgeons in near-complete tumor removal to increase survival and reduce neurological deficits.

(http://thejns.org/doi/abs/10.3171/2013.11.FOCUS13497)

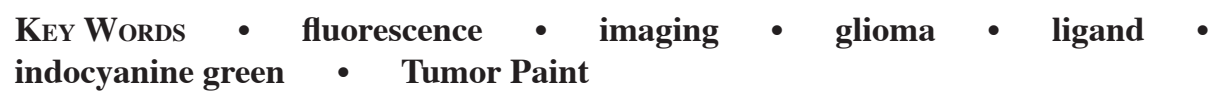

$\mathrm{G}$ LIOMAS are the deadliest and commonest tumors of the CNS. The current 18 -month survival rate of glioblastoma patients treated with complete resection ranges from $15 \%$ to $34 \%$, making glioma one of the most lethal tumors. ${ }^{6}$ Additionally, these tumors' deleterious effects are visible in the patient's quality of life, their family, and the health care system, with a mean survival of 14 months. Gliomas have a reported incidence of 10 per 100,000 per year and result in 13,000 deaths per year, accounting for more years lost than any other tumor. ${ }^{11}$ Although multiple options such as chemotherapy

Abbreviations used in this paper: $\mathrm{CCD}=$ charge-coupled device; $\mathrm{CTX}=$ chlorotoxin; $\mathrm{HD}=$ high-definition; $\mathrm{ICG}=$ indocyanine green; NIR = near-infrared. and radiation therapy are available for the treatment of gliomas, due to the inability of chemotherapeutic agents to cross the blood-brain barrier and the ineffectiveness as well as adverse effects of radiation therapy, resection remains the primary treatment. ${ }^{10,13,27}$ The aggressiveness of tumor surgery and extent of resection in prolonging patient survival is controversial; ${ }^{11,12,18}$ however, there is evidence that minimizing residual tumor will decrease the likelihood of recurrence. ${ }^{3,30}$ There are multiple publications over the past couple of decades that have demonstrated that surgical extent is the single most important determinant of outcome and the most important predictor of longer survival. ${ }^{4,7,28}$ Evidence also suggests that more complete resection of gliomas correlates with a higher progression-free interval and may prolong life expec- 


\section{P. V. Butte et al.}

tancy. ${ }^{11,12,18}$ One of the primary challenges of surgical removal of brain tumors is to identify and visualize the tumor tissue separate from the normal cortex. Balancing aggressive resection against the necessity to limit resection of surrounding normal brain tissue is the core goal of brain tumor surgery. Intraoperative fluorescence-guided resection can facilitate this goal by defining the location of the tumor and delineating the brain-tumor border. ${ }^{26}$ Thus, newer technologies are needed that can accurately distinguish between the tumor and normal brain and guide tumor resection in real time.

\section{Tumor-Specific Ligand}

Targeting molecules such as antibodies and peptides are being investigated as tumor-specific ligands. One such ligand is chlorotoxin (CTX), a 36-amino acid peptide initially isolated from the venom of the scorpion Leiurus quinquestriatus. The peptide has demonstrated specificity as a tumor-targeting agent in a variety of formats, including radiolabeled, conjugated to fluorescent tags, or incorporated into nanoparticles. ${ }^{1,29}$ All clinical trials to date conducted with CTX indicate that it has negligible toxicity in humans. These unique properties make CTX attractive as a targeted imaging agent for cancer. In transgenic and xenograft mouse models of glioma, medulloblastoma, prostate cancer, sarcoma, and colorectal cancer, a CTX-Cy5.5 conjugate ("Tumor Paint") was shown to bind to both primary tumor and metastatic lesions. ${ }^{37}$ Subsequent work with CTX conjugated to a variety of near-infrared (NIR) fluorophores confirmed and extended these results, supporting the peptide's specificity and breadth of tumor recognition. ${ }^{20} \mathrm{BLZ}-100$ is a CTX-indocyanine green (ICG) conjugate that is being advanced toward human clinical trials.

In the past few years there has been an increased interest in NIR light fluorescence imaging. ${ }^{32}$ Near-infrared contrast agents such as ICG offer well-recognized advantages over ultraviolet or visible light imaging. These include better tissue penetration of incident light, less scatter of emitted photons, and low tissue autofluorescence. ${ }^{32}$ There are multiple imaging systems that have been developed by both academic and industrial groups. $5,8,9,17,19,21,22,24,25,31,33,38,39$ There are separate systems for open surgeries, laparoscopic surgeries, and robot-assisted surgeries that vary based on specific challenges. Some of the important criteria for developing a new imaging system are based on concentration of the fluorophores, type of tissue due to varying tissue penetration depths, ergonomics, and cost. Additionally, NIR imaging systems have to optimize the excitation light fluence, and there is a choice between coherent and incoherent light sources. Incoherent light sources are easier to work with, require fewer precautions for skin and eye exposure, and face fewer regulatory hurdles, but require high energy to produce adequate fluorescence. Coherent sources (lasers) are usually restricted due to skin and eye exposure to $10-25 \mathrm{~mW} / \mathrm{cm}^{2}$ to negate the need for protective glasses, ${ }^{32}$ but if the photobleaching effect on the fluorophore is absent at higher energies and the use of protective glasses is not an issue during the surgery, it may be possible to use higher energy. Considering these factors, we would like to report development of a "proof-of-concept" intraopera- tive NIR imaging system to detect a novel tumor ligand attached to an NIR dye (ICG) for use in brain tumor surgeries.

\section{Methods}

\section{Fluorescence Imaging System}

To address the need for a clinically relevant tool for NIR fluorescence-guided resection of tumors, we developed a camera system that simultaneously acquires both white light and NIR images and combines these images via superimposition on a high-definition (HD) video monitor. The proof-of-concept system consists of a dual HD charge-coupled device (CCD) camera that splits incident light into 2 pathways, one for white light and the other for NIR light (AD-130GE, 1/3", $1296 \times 966,31 \mathrm{fps,}$ GigE; JAI, Inc.) (Fig. 1).

A fixed focal length lens (16-mm VIS-NIR Compact Fixed Focal Length Lens, Edmund Optics) was attached using a C-mount. A thin filter holder (NT56-353, CMount Thin Lens Mount, 25/25.4-mm diameter, Edmund Optics) was attached in front of the lens, which housed a $785-\mathrm{nm}$ notch filter to filter out the excitation light from the return image (785-nm StopLine single-notch filter, NF03-785E-25, Semrock). Near-infrared excitation is provided via a 785-nm laser diode (LD785-SH300, Thorlabs), while white light is provided through a commercially available xenon light source (Storz). The camera acquires both white light and NIR fluorescence images. These are captured via a GigE interface to a computer. The NIR images are given a transparency mask, given a false color, and added to the white light image. The resultant HD-quality images superimposed with fluorescent maps of BLZ-100 distribution can be used to direct intraoperative detection and resection of tumor. The camera system can detect BLZ-100 in nanomolar concentrations. Since the images are collected digitally, the detection sensitivity can be adjusted utilizing analog and digital gain and threshold to maximize detection in the NIR, as well as providing artificial color maps.

\section{BLZ-100}

The CTX peptide was synthesized using standard solid-phase methods. After refolding, the peptide was conjugated to an active ICG derivative using standard NHS ester chemistry. The conjugate underwent high performance liquid chromatography purification.

\section{Intralipid Studies}

To test the sensitivity of the instrument to detect BLZ100 , a range of BLZ-100 concentrations were prepared in 5\% Intralipid emulsion (Sigma Aldrich). The BLZ-100 concentrations tested were $1 \mu \mathrm{M}, 500 \mathrm{nM}, 50 \mathrm{nM}$, and 50 pM in 5\% Intralipid emulsion, and $20 \%$ Intralipid emulsion was tested with no BLZ-100 as a control. The laser fluence was varied to determine the optimal fluence rate for each BLZ-100 concentration. The following fluence rates were tested: $5,10,15,20,30$, and $40 \mathrm{~mW} / \mathrm{cm}^{2}$. The analog again on the CCD was set at $0 \mathrm{~dB}, 6 \mathrm{~dB}$, and 12 $\mathrm{dB}$, and the fluorescence intensity was noted. 


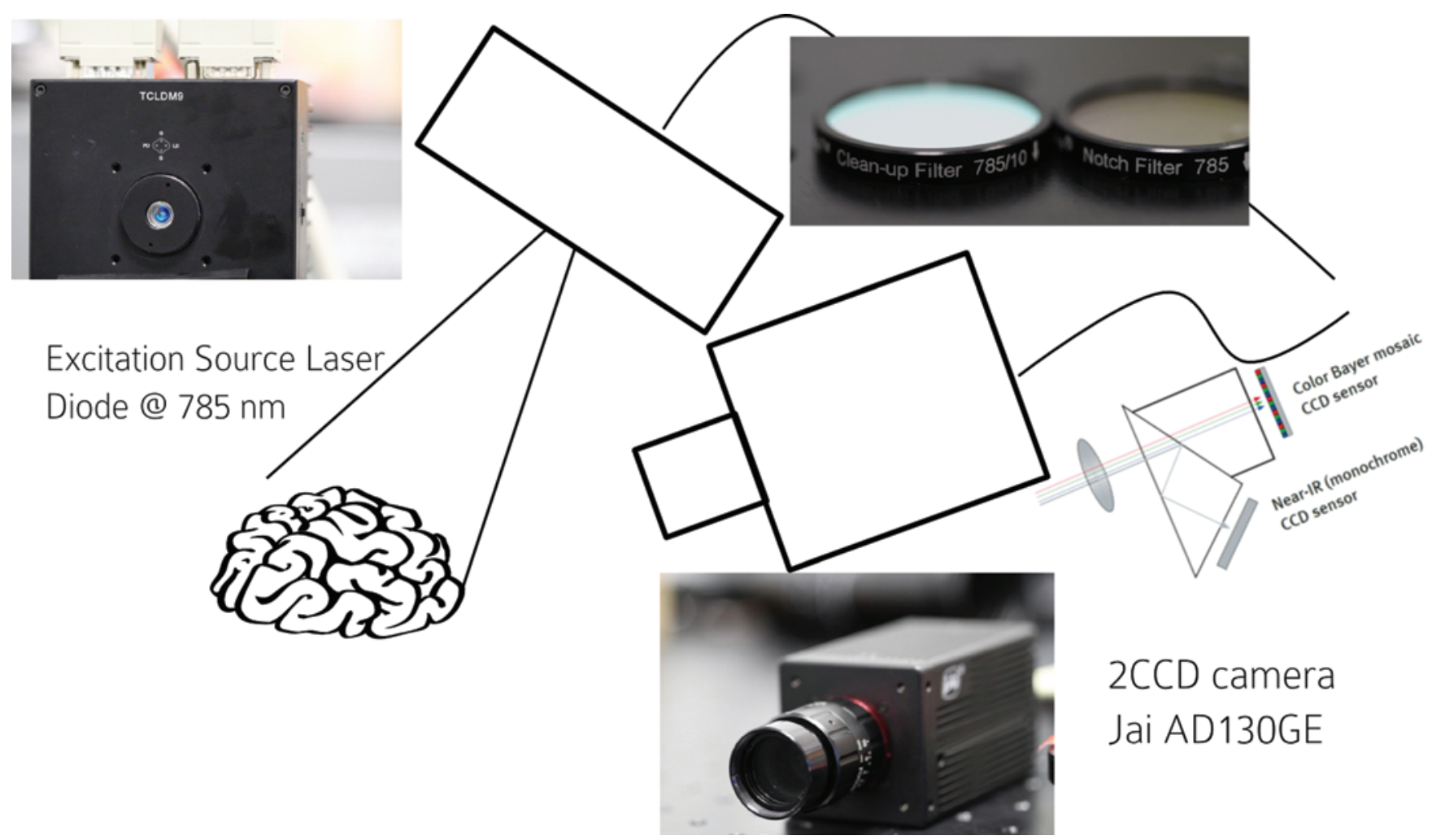

FIG. 1. Schematic showing the prototype NIR imaging system.

\section{Intracranial Tumors in Animals}

BLZ-100 was tested in mice implanted with human glioblastoma cells along with ICG as control. The mice were treated according to the approved Cedars-Sinai Medical Center Institutional Animal Care and Use Committee protocols. Athymic mice (NCr-nu/nu homozygous) were obtained from the National Cancer Institute. Human glioblastoma cells (LN229) were stereotactically implanted at $5 \times 10^{5}$ cells/mouse into the right basal ganglia field of mice under ketamine and dexmedetomidine intraperitoneal anesthesia, as reported previously. ${ }^{14}$ The animals were closely monitored and used for the experiment about 6 weeks postinoculation, when the tumors had reached maximum size.

In 1 mouse that was under inhalation isoflurane anesthesia, vascular contrast dye (20 nmol ICG) was injected intravenously once via the tail vein using a 30-gauge needle on a $1-\mathrm{ml}$ syringe at a rate of $50 \mu \mathrm{l}$ over 3 seconds. Another mouse was injected in a similar manner with 20 nmol of BLZ-100. One set of mice received phosphatebuffered saline as control. After drug administration, the mice were observed for several hours for any physical symptoms or behavioral changes. Forty-eight hours after injection, animals were anesthetized via intraperitoneal injection with a combination of ketamine and dexmedetomidine and were euthanized via cervical dislocation.

The mice were then placed securely in the stereotactic apparatus, and a $10-\mathrm{mm}$ midline incision was made in the scalp, as for the tumor cell inoculation. A craniectomy was then performed over the tumor site. Once the skull was removed, direct in situ tumor imaging was performed, after which the brain and other organs were removed for individual imaging and processing. Direct imaging of the tumor site was done after euthanasia.

\section{Results}

\section{Intralipid Study}

To quantitate the sensitivity of the CIRCAM for BLZ-100, a series of dilutions were tested in Intralipid emulsion, which mimics the light scattering and absorption properties of tissue. At $1 \mu \mathrm{M}$ BLZ-100, the CCD was able to record fluorescence at all the fluence rates and all the CCD gains. The imaging system was able to record fluorescence at the lowest excitation fluence of $5 \mathrm{~mW} / \mathrm{cm}^{2}$ and with no analog gain on the CCD (Fig. 2).

The fluorescence increased when the concentration of BLZ-100 was decreased to $500 \mathrm{nM}(0.5 \mu \mathrm{M})$ as observed in Figs. 3 and 4 (6 dB and $12 \mathrm{~dB}$ gain). This is believed to be due to reduction in fluorescence quenching.

When BLZ-100 was diluted to $50 \mathrm{nM}$ of concentration, the fluorescence was difficult to discern at $0-\mathrm{dB}$ gain on CCD, but when the analog gain of the CCD was increased to $6 \mathrm{~dB}$, fluorescence could be seen at $20 \mathrm{~mW} /$ $\mathrm{cm}^{2}$ of laser fluence. Although the samples demonstrated visible fluorescence when CCD gain was set at $12 \mathrm{~dB}$ at all the excitation energies, there was a significant increase in the CCD noise (Fig. 4). At $50 \mathrm{pM}$, fluorescence from BLZ-100 was barely detectable at $40 \mathrm{~mW} / \mathrm{cm}^{2}$ with 12 $\mathrm{dB}$ of gain. Finally, 20\% Intralipid solution was tested, which showed no fluorescence at any excitation energy and CCD gain (Fig. 4). 


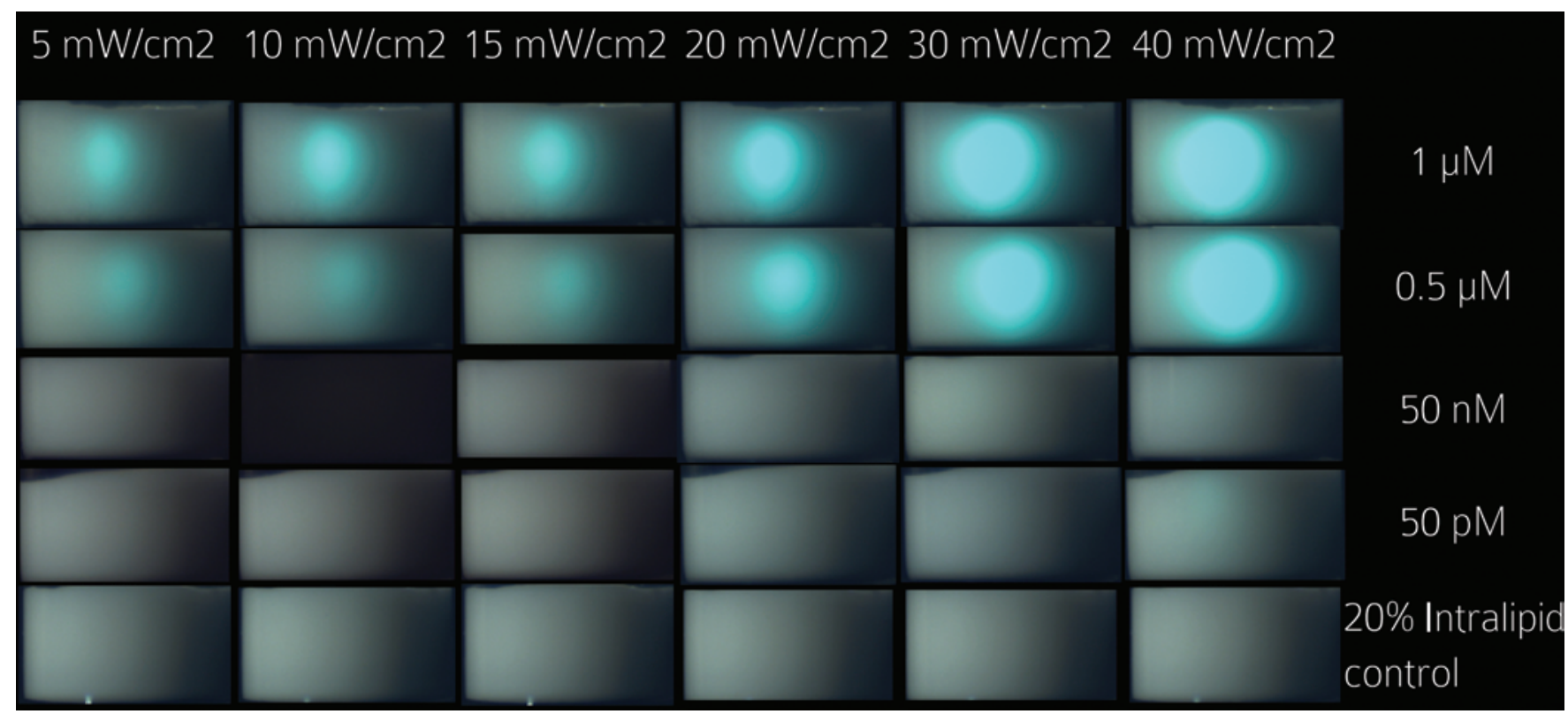

FIG. 2. Fluorescence from BLZ-100 mixed in $5 \%$ Intralipid emulsion was recorded at varying laser fluence with 0-dB analog gain. As seen in the image, there is no discernible fluorescence at a low concentration of $0.5 \mu \mathrm{m}$. The change in the fluorescence area was due to Gaussian profile of the laser diode emission.

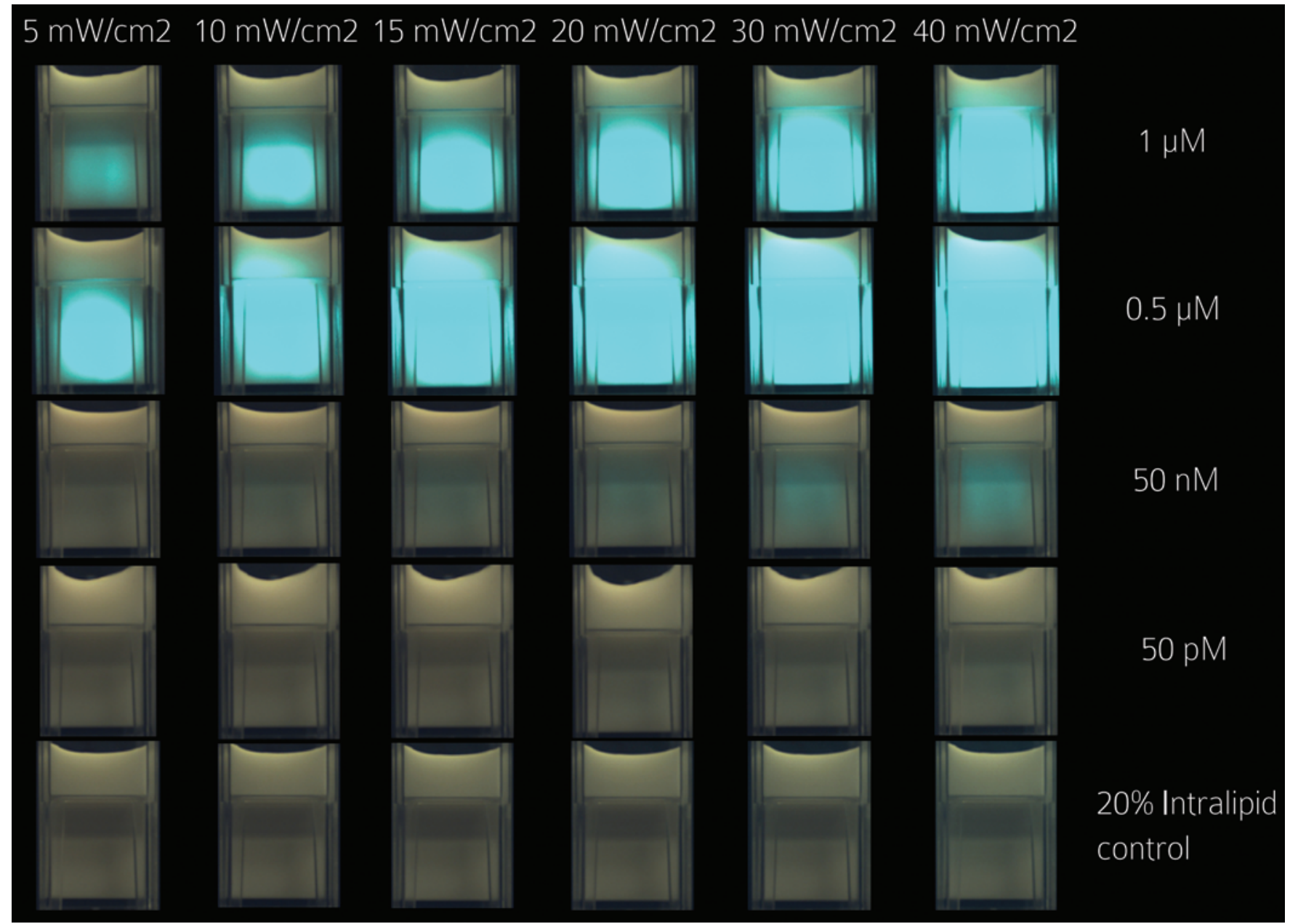

FIG. 3. Fluorescence from BLZ-100 mixed in $5 \%$ Intralipid emulsion was recorded at varying laser fluence with 6-dB analog gain. As seen in the results with the analog gain, it is possible to visualize BLZ-100 down to $50 \mathrm{nM}$ concentration at fluence of $20 \mathrm{~mW} / \mathrm{cm}^{2}$. 


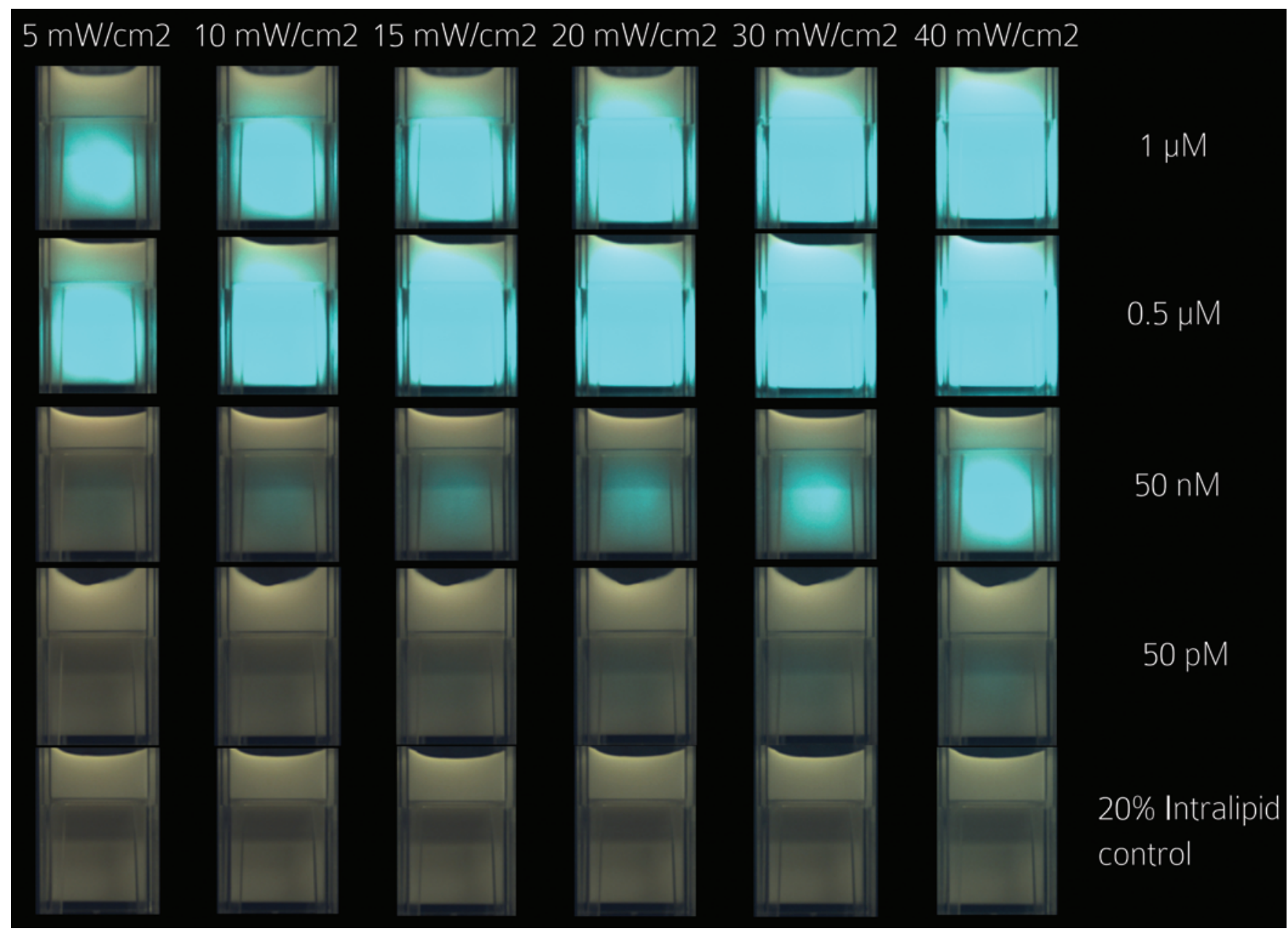

FIG. 4. Fluorescence from BLZ-100 mixed in 5\% Intralipid emulsion was recorded at varying laser fluence with 12-dB analog gain. As seen in the image, ICG fluorescence was visible down to $50 \mathrm{pM}$ with a high signal-to-noise ratio when excited using 40 $\mathrm{mW} / \mathrm{cm}^{2}$.

\section{In Vivo Study (Brain)}

To demonstrate effective discrimination between BLZ100-labeled tumors and surrounding normal brain tissue, mice bearing orthotopic glioma tumors were treated with BLZ-100 and were imaged 48 hours later. The mice injected with BLZ-100 showed a significant fluorescence from the glioma (Figs. 5 and 6), where there was no fluorescence observed from the mice injected with plain ICG. BLZ100 accumulated in the glioma. Additionally, fluorescence from BLZ-100 was able to show a deeper metastasis (Fig. $5)$. The organs harvested from the mice were also recorded for fluorescence (Fig. 7). There was no visible fluorescence from the stomach, heart, lung, and skin. The kidney and liver showed detectable amounts of BLZ-100.

\section{Discussion}

Near-Infrared Imaging and Tumor Ligands

Compared with intraoperative x-ray fluoroscopy, ultrasonography, CT, and MRI, targeted NIR imaging affords the combination of tumor specificity, low cost, simplicity, and safety, without exposing patients and personnel to ion- izing radiation. ${ }^{32}$ The NIR technique involves the use of an imaging system or device along with a contrast fluorophore. ${ }^{2,32}$ Multiple optical imaging systems are currently out on the market, and pertinent contrast agents include methylene blue and ICG. ${ }^{2,32}$ The technology has seen clini-
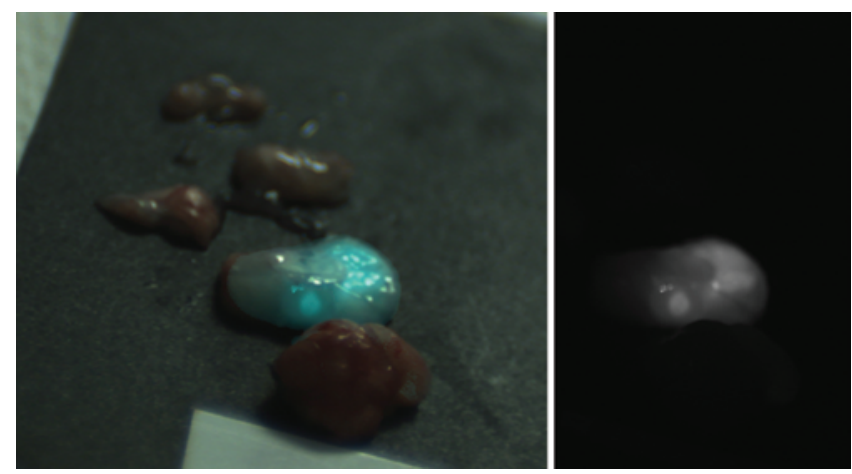

FIG. 5. Left: White light image overlaid with an NIR image of a brain slice with implanted glioma. Right: Image from the NIR channel, CCD gain was set at $0 \mathrm{~dB}$, and excitation fluence was $40 \mathrm{~mW} / \mathrm{cm}^{2}$. Notice the satellite metastatic lesion separate from the main tumor body. Due to high penetration depth of the excitation light, NIR imaging is able to visualize deep tumors, not seen by visual inspection. 

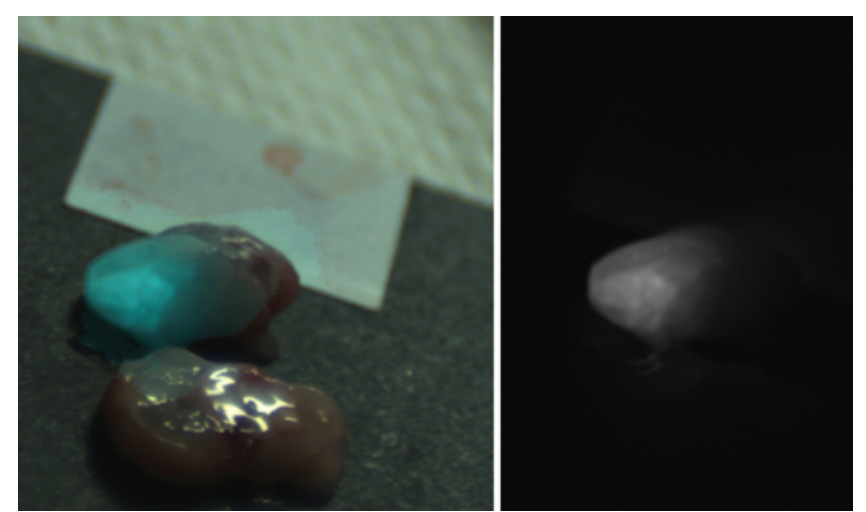

FIG. 6. Left: White light image overlaid with an NIR image of sections through the brain sample implanted with human glioma cells. Right: A separate NIR channel.

cal applications in multiple fields including laparoscopy, hepatology, coronary artery surgery, vascular surgery, and surgical oncology. ${ }^{2,32}$ In particular, there has been a lot of interest in NIR imaging using ICG, with reported uses in detection of breast cancer, melanoma, otolaryngological malignancies, and liver metastasis. ${ }^{15,16,22,23,33-36}$ Indocyanine green (ICG) is a water-soluble molecule that has been used in angiographic, cardiac, hepatic, and oncological applications. ${ }^{3,26}$ Its minimal side effects and positive safety profile have made it an increasingly used compound in multiple disciplines. In neurosurgery, it has been demonstrated to outline tumor boundaries and differentiate low-grade from high-grade tumor cells. ${ }^{3}$ It has also been routinely used to aid aneurysm and arteriovenous malformation surgery through ICG video angiography. ${ }^{3}$
Recently, NIR technology has been used alongside fluorescent tumor ligands. These cancer-targeted molecules can be used to specifically isolate tumors for resection, and combining them with fluorescent molecules and NIR technology can make them even more powerful tools. The currently available NIR imaging systems are optimized for high-volume and high-concentration ICG for visualizing vasculature or sentinel lymph nodes. Additionally, the imaging systems are bulky and many are as big as the operating microscopes. An important criterion for us in designing the system for brain tumor imaging was to have a small profile so as to allow the surgeon to use the operating microscope separate from the NIR imaging system. Most of the traditional NIR systems available use 2 separate sensors for visible and NIR channels. The NIR light is redirected separate from the visible light using a beam splitter. Although this framework allows for the use of a separate high-sensitivity infrared optimized camera, it also adds to the weight and size of the system. To avoid this problem we have designed a new system that uses the same sensor for both visible and infrared channels. However, to test the hypothesis that a single sensor with the NIR filter removed can have enough sensitivity to record fluorescence from the brain tumor, we used the JAI AD-130GE camera, which uses identical CCDs for both infrared and visible channels. From our initial results we can report that BLZ-100 fluorescence can be recorded by a CCD, which is neither cooled nor intensified.

We also chose to use laser excitation instead of an incoherent light source such as xenon lamps. This allowed us to use a very narrow notch filter instead of a broad long-pass filter to remove the excitation light and collect

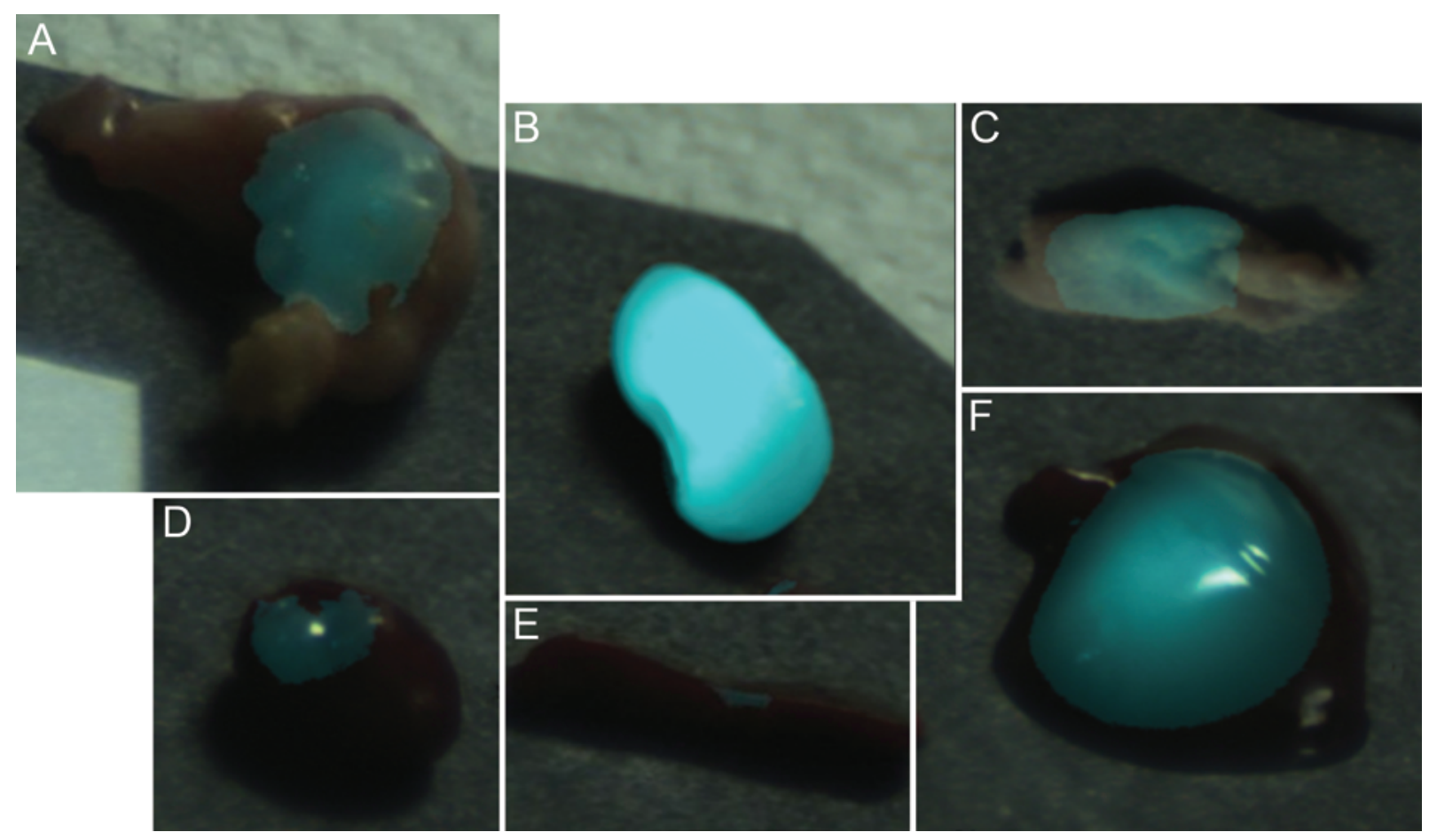

FIG. 7. White light images overlaid with NIR images of organs showing fluorescence from the residual BLZ-100. There is very low or no discernible fluorescence from stomach (A), skin (C), heart (D), and aorta (E), while the kidney (B) and liver (F) show significant fluorescence as ICG is excreted through the kidney. 
maximum fluorescence. Laser light is also more efficient in generating fluorescence from the ICG than incoherent light. Thus, by optimizing both the excitation as well as fluorescence light paths, we were able to achieve high sensitivity with very low noise.

\section{Conclusions}

We have demonstrated as a proof-of-concept that it is possible to use a standard $C C D$ for recording fluorescence from brain tumor using BLZ-100, a tumor ligand conjugated to ICG. This will enable us to reduce the size and cost of the imaging system. The use of a narrow-band laser at the peak absorption of ICG allows us to use low excitation fluence, and use of a narrow notch filter in front of the sensor will allow us to gather maximum fluorescence.

\section{Acknowledgment}

We thank Dr. David Kittle for his help with the arrangement and formatting of the images.

\section{Disclosure}

The authors thank Stacy Hansen and Disha Sahetya from Blaze Bioscience, Inc., Seattle, for providing the BLZ-100 samples. The study was internally funded by the Department of Neurosurgery at Cedars-Sinai Medical Center. Dr. Mamelak has ownership in Teal Light Surgical. Dr. Parrish-Novak is an employee of Blaze Bioscience, Inc.

Author contributions to the study and manuscript preparation include the following. Conception and design: Butte, Mamelak, Parrish-Novak. Acquisition of data: Butte, Gangalum, Chesnokova. Analysis and interpretation of data: Butte. Drafting the article: Butte, Drazin, Shweikeh, Gangalum. Reviewed submitted version of manuscript: Mamelak, Parrish-Novak. Administrative/technical/ material support: Mamelak, Parrish-Novak, Gangalum, Chesnokova, Ljubimova, Black. Study supervision: Mamelak, Parrish-Novak.

\section{References}

1. Akcan M, Stroud MR, Hansen SJ, Clark RJ, Daly NL, Craik DJ, et al: Chemical re-engineering of chlorotoxin improves bioconjugation properties for tumor imaging and targeted therapy. J Med Chem 54:782-787, 2011

2. Alander JT, Kaartinen I, Laakso A, Pätilä T, Spillmann T, Tuchin VV, et al: A review of indocyanine green fluorescent imaging in surgery. Int J Biomed Imaging 2012:940585, 2012

3. Behbahaninia M, Martirosyan NL, Georges J, Udovich JA, Kalani MY, Feuerstein BG, et al: Intraoperative fluorescent imaging of intracranial tumors: a review. Clin Neurol Neurosurg 115:517-528, 2013

4. Berger MS: Malignant astrocytomas: surgical aspects. Semin Oncol 21:172-185, 1994

5. Borofsky MS, Gill IS, Hemal AK, Marien TP, Jayaratna I, Krane LS, et al: Near-infrared fluorescence imaging to facilitate super-selective arterial clamping during zero-ischaemia robotic partial nephrectomy. BJU Int 111:604-610, 2013

6. Brandes AA, Tosoni A, Franceschi E, Reni M, Gatta G, Vecht C: Glioblastoma in adults. Crit Rev Oncol Hematol 67:139152,2008

7. Byar DP, Green SB, Strike TA: Prognostic factors for malignant glioma, in Walker MD (ed): Oncology of the Nervous System. Boston: Martinus Nijhoff, 1983, pp 379-395

8. Cahill RA, Anderson M, Wang LM, Lindsey I, Cunningham C, Mortensen NJ: Near-infrared (NIR) laparoscopy for intraoperative lymphatic road-mapping and sentinel node iden- tification during definitive surgical resection of early-stage colorectal neoplasia. Surg Endosc 26:197-204, 2012

9. Crane LM, Themelis G, Pleijhuis RG, Harlaar NJ, Sarantopoulos A, Arts HJ, et al: Intraoperative multispectral fluorescence imaging for the detection of the sentinel lymph node in cervical cancer: a novel concept. Mol Imaging Biol 13:1043-1049, 2011 (Erratum in Mol Imaging Biol 13:1050, 2011)

10. Ducray F, Dutertre G, Ricard D, Gontier E, Idbaih A, Massard C: [Advances in adults' gliomas biology, imaging and treatment.] Bull Cancer 97:17-36, $2010(\mathrm{Fr})$

11. Eljamel MS: Fluorescence image-guided surgery of brain tumors: explained step-by-step. Photodiagn Photodyn Ther 5: 260-263, 2008

12. Eljamel MS, Goodman C, Moseley H: ALA and Photofrin fluorescence-guided resection and repetitive PDT in glioblastoma multiforme: a single centre Phase III randomised controlled trial. Lasers Med Sci 23:361-367, 2008

13. Fazekas JT: Treatment of grades I and II brain astrocytomas. The role of radiotherapy. Int J Radiat Oncol Biol Phys 2: 661-666, 1977

14. Fujita M, Lee BS, Khazenzon NM, Penichet ML, Wawrowsky KA, Patil R, et al: Brain tumor tandem targeting using a combination of monoclonal antibodies attached to biopoly(beta-Lmalic acid). J Control Release 122:356-363, 2007

15. Gilmore DM, Khullar OV, Colson YL: Developing intrathoracic sentinel lymph node mapping with near-infrared fluorescent imaging in non-small cell lung cancer. J Thorac Cardiovasc Surg 144:S80-S84, 2012

16. Gilmore DM, Khullar OV, Jaklitsch MT, Chirieac LR, Frangioni JV, Colson YL: Identification of metastatic nodal disease in a phase 1 dose-escalation trial of intraoperative sentinel lymph node mapping in non-small cell lung cancer using near-infrared imaging. J Thorac Cardiovasc Surg 146:562570,2013

17. Gotoh K, Yamada T, Ishikawa O, Takahashi H, Eguchi H, Yano M, et al: A novel image-guided surgery of hepatocellular carcinoma by indocyanine green fluorescence imaging navigation. J Surg Oncol 100:75-79, 2009

18. Hefti M, von Campe G, Moschopulos M, Siegner A, Looser H, Landolt $\mathrm{H}$ : 5-aminolevulinic acid induced protoporphyrin IX fluorescence in high-grade glioma surgery: a one-year experience at a single institution. Swiss Med Wkly 138:180-185, 2008

19. Hirche C, Engel H, Kolios L, Cognie J, Hünerbein M, Lehnhardt M, et al: An experimental study to evaluate the fluobeam 800 imaging system for fluorescence-guided lymphatic imaging and sentinel node biopsy. Surg Innov 20:516-523, 2013

20. Mamelak AN, Jacoby DB: Targeted delivery of antitumoral therapy to glioma and other malignancies with synthetic chlorotoxin (TM-601). Expert Opin Drug Deliv 4:175-186, 2007

21. Marano A, Priora F, Lenti LM, Ravazzoni F, Quarati R, Spinoglio G: Application of fluorescence in robotic general surgery: review of the literature and state of the art. World J Surg 37:2800-2811, 2013

22. Mieog JS, Hutteman M, van der Vorst JR, Kuppen PJ, Que I, Dijkstra J, et al: Image-guided tumor resection using real-time near-infrared fluorescence in a syngeneic rat model of primary breast cancer. Breast Cancer Res Treat 128:679-689, 2011

23. Mieog JS, Troyan SL, Hutteman M, Donohoe KJ, van der Vorst JR, Stockdale A, et al: Toward optimization of imaging system and lymphatic tracer for near-infrared fluorescent sentinel lymph node mapping in breast cancer. Ann Surg Oncol 18:2483-2491, 2011

24. Moroga T, Yamashita S, Tokuishi K, Miyawaki M, Anami K, Yamamoto S, et al: Thoracoscopic segmentectomy with intraoperative evaluation of sentinel nodes for stage I non-small cell lung cancer. Ann Thorac Cardiovasc Surg 18:89-94, 2012

25. Phillips BT, Lanier ST, Conkling N, Wang ED, Dagum AB, Ganz JC, et al: Intraoperative perfusion techniques can accu- 
rately predict mastectomy skin flap necrosis in breast reconstruction: results of a prospective trial. Plast Reconstr Surg 129:778e-788e, 2012

26. Pogue BW, Gibbs-Strauss S, Valdés PA, Samkoe K, Roberts DW, Paulsen KD: Review of neurosurgical fluorescence imaging methodologies. IEEE J Sel Top Quantum Electron 16: 493-505, 2010

27. Robins HI, Lassman AB, Khuntia D: Therapeutic advances in malignant glioma: current status and future prospects. Neuroimaging Clin N Am 19:647-656, 2009

28. Sanai N, Berger MS: Glioma extent of resection and its impact on patient outcome. Neurosurgery 62:753-766, 2008

29. Stroud MR, Hansen SJ, Olson JM: In vivo bio-imaging using chlorotoxin-based conjugates. Curr Pharm Des 17:43624371, 2011

30. Stummer W, Reulen HJ, Meinel T, Pichlmeier U, Schumacher W, Tonn JC, et al: Extent of resection and survival in glioblastoma multiforme: identification of and adjustment for bias. Neurosurgery 62:564-576, 2008

31. Themelis G, Yoo JS, Soh KS, Schulz R, Ntziachristos V: Realtime intraoperative fluorescence imaging system using lightabsorption correction. J Biomed Opt 14:064012, 2009

32. Vahrmeijer AL, Hutteman M, van der Vorst JR, van de Velde CJ, Frangioni JV: Image-guided cancer surgery using nearinfrared fluorescence. Nat Rev Clin Oncol 10:507-518, 2013

33. van der Poel HG, Buckle T, Brouwer OR, Valdés Olmos RA, van Leeuwen FW: Intraoperative laparoscopic fluorescence guidance to the sentinel lymph node in prostate cancer patients: clinical proof of concept of an integrated functional imaging approach using a multimodal tracer. Eur Urol 60:826833, 2011

34. van der Vorst JR, Hutteman M, Mieog JS, de Rooij KE, Kaijzel EL, Löwik CW, et al: Near-infrared fluorescence imaging of liver metastases in rats using indocyanine green. J Surg Res 174:266-271, 2012

35. van der Vorst JR, Schaafsma BE, Verbeek FP, Swijnenburg RJ, Tummers QR, Hutteman M, et al: Intraoperative near-infrared fluorescence imaging of parathyroid adenomas with use of lowdose methylene blue. Head Neck [epub ahead of print], 2013

36. van der Vorst JR, Vahrmeijer AL, Hutteman M, Bosse T, Smit VT, van de Velde CJ, et al: Near-infrared fluorescence imaging of a solitary fibrous tumor of the pancreas using methylene blue. World J Gastrointest Surg 4:180-184, 2012

37. Veiseh M, Gabikian P, Bahrami SB, Veiseh O, Zhang M, Hackman RC, et al: Tumor paint: a chlorotoxin:Cy5.5 bioconjugate for intraoperative visualization of cancer foci. Cancer Res 67:6882-6888, 2007

38. Yamashita S, Tokuishi K, Anami K, Miyawaki M, Moroga T, Kamei M, et al: Video-assisted thoracoscopic indocyanine green fluorescence imaging system shows sentinel lymph nodes in non-small-cell lung cancer. J Thorac Cardiovasc Surg 141:141-144, 2011

39. Yamauchi K, Nagafuji H, Nakamura T, Sato T, Kohno N: Feasibility of ICG fluorescence-guided sentinel node biopsy in animal models using the HyperEye Medical System. Ann Surg Oncol 18:2042-2047, 2011

Manuscript submitted October 18, 2013.

Accepted November 26, 2013.

Please include this information when citing this paper: DOI: 10.3171/2013.11.FOCUS13497.

Address correspondence to: Pramod V. Butte, M.B.B.S., Ph.D., Department of Neurosurgery, Cedars-Sinai Medical Center, 127 S. San Vicente Blvd., Ste. A8224, Los Angeles, CA 90048. email: pramod.butte@cshs.org. 